\title{
Focal choroidal excavation in eyes with central serous chorioretinopathy.
}

\section{AUTHOR(S):}

Ellabban, Abdallah A; Tsujikawa, Akitaka; Ooto, Sotaro; Yamashiro, Kenji; Oishi, Akio; Nakata, Isao; Miyake, Masahiro; ... Yoshitake, Shin; Takahashi, Ayako; Yoshimura, Nagahisa

\section{CITATION:}

Ellabban, Abdallah A ... [et al]. Focal choroidal excavation in eyes with central serous chorioretinopathy.. American journal of ophthalmology 2013, 156(4): 673-683.e1

\section{ISSUE DATE:}

2013-10

URL:

http://hdl.handle.net/2433/179295

\section{RIGHT:}

(C) 2013 Elsevier Inc:; This is not the published version. Please cite only the published version.; この論文は出版社版でありません。引用の際に は出版社版をご確認ご利用ください。 


\section{ABSTRACT}

Purpose: To study the prevalence and three-dimensional (3-D) tomographic features of focal choroidal excavations in eyes with central serous chorioretinopathy (CSC) using swept-source optical coherence tomography (OCT).

Design: Prospective, cross-sectional study.

Methods: We examined 116 consecutive eyes with CSC with a prototype 3-D swept-source OCT. 3-D images of the shape of the macular area, covering $6 \times 6 \mathrm{~mm}^{2}$, were reconstructed by segmentation of the outer surface of the retinal pigment epithelium (RPE).

Results: The 3-D swept-source OCT detected focal choroidal excavations in 9 eyes (7.8\%). The 3-D scanning protocol, coupled with en-face scans, allowed for clear visualization of the excavation morphology. In 5 eyes with focal excavations, unusual choroidal tissue was found beneath the excavation, bridging the bottom of the excavation and the outer choroidal boundary. Additionally, three of those five eyes showed a suprachoroidal space below the excavation, as if the outer choroidal boundary is pulled inward by this bridging tissue. The focal choroidal excavations were located within fluorescein leakage points and areas of choroidal hyperpermeability. Eyes with focal choroidal excavations were more myopic (-4.42 \pm 2.92 diopters) than eyes without excavations $(-0.27 \pm 1.80, P=.001)$. Subfoveal choroidal thickness was significantly thinner $(301.3 \pm 60.1 \mu \mathrm{m})$ in eyes with focal excavations than in eyes without the excavations (376.6 $\pm 104.8 \mu \mathrm{m}, P=.036)$.

CONCLUSIONS: Focal choroidal excavations were present in $7.8 \%$ of eyes with CSC. In these eyes, focal choroidal excavations may have formed from RPE retraction caused by focal scarring of choroidal connective tissue. 


\title{
FOCAL CHOROIDAL EXCAVATION IN EYES WITH CENTRAL SEROUS CHORIORETINOPATHY
}

\author{
ABDALLAH A. ELLABBAN,${ }^{1,2}$ AKITAKA TSUJIKAWA, ${ }^{1}$ SOTARO OOTO, ${ }^{1} \mathrm{KENJI}$ \\ YAMASHIRO, ${ }^{1}$ AKIO OISHI, ${ }^{1}$ ISAO NAKATA, ${ }^{1}$ MASAHIRO MIYAKE, ${ }^{1}$ YUMIKO \\ AKAGI-KURASHIGE, ${ }^{1}$ NAOKO UEDA-ARAKAWA, ${ }^{1}$ SHIGETA ARICHIKA, ${ }^{1}$ SHIN \\ YOSHITAKE,${ }^{1}$ AYAKO TAKAHASHI, ${ }^{1}$ AND NAGAHISA YOSHIMURA ${ }^{1}$ \\ 1'Department of Ophthalmology and Visual Sciences, Kyoto University Graduate School \\ of Medicine, Kyoto, Japan; ${ }^{2}$ Department of Ophthalmology, Suez Canal University, \\ Faculty of Medicine, Ismailia, Egypt.
}

Supplemental Material available at AJO.com

Inquiries to Akitaka Tsujikawa, Department of Ophthalmology and Visual Sciences, Kyoto University Graduate School of Medicine, Sakyo-ku, Kyoto 606-8507, Japan; e-mail: tujikawa@kuhp.kyoto-u.ac.jp

Short title: FOCAL CHOROIDAL EXCAVATION 


\section{INTRODUCTION}

Central serous chorioretinopathy (CSC) is characterized by serous retinal detachment in the macular area, as confirmed by leakage on fluorescein angiography (FA). In eyes with CSC, indocyanine green angiography (ICGA) often shows choroidal vascular abnormalities such as choroidal filling delay, dilated vasculature, choroidal hyperpermeability, and punctate hyperfluorescent spots. ${ }^{1-9}$ Recent advances in choroidal imaging with optical coherence tomography (OCT), coupled with the enhanced depth imaging technique,$^{10}$ have revealed choroidal thickening in eyes with CSC and such thickening deceased after the treatment with photodynamic therapy. ${ }^{11-17}$ These observations suggest that the underlying pathogenesis of CSC is primarily related to functional abnormalities of the choroidal vasculature.

On the basis of OCT imaging, focal choroidal excavation ${ }^{18-24}$ was recently reported as a localized area of excavation within the submacular choroid. Focal choroidal excavation was first reported to occur in otherwise healthy eyes without any ocular comorbidities. ${ }^{22,23}$ However, later reports showed that focal choroidal excavations are associated with vision-threatening diseases such as CSC, polypoidal choroidal vasculopathy, and choroidal neovascularization. ${ }^{18-20}$ Recently, Margolis and associates ${ }^{20}$ reported a case of focal choroidal excavation in an eye with CSC and suggested that there could be an association between the two conditions. However, mechanisms underlying this unusual excavation remain unknown. In addition, most previously reported cases of focal choroidal excavations were examined with unidirectional OCT scans $^{18-23}$ and little information is available on the 3-dimensional (3-D) shape of the excavations. So far, the prevalence of focal choroidal excavation remains unknown because previous reports are either single case reports or small case series.

Recent advances in OCT technology include the utilization of a swept-source laser as the light source. ${ }^{25-27}$ The swept-source OCT with a longer wavelength light source provides better views of the choroid because of improved light penetration into the choroid. Additionally, the tunable laser source of the swept-source OCT shows lower signal decay with depth, further improving the visibility of choroidal details. Furthermore, the higher imaging speed allows for dense scanning and subsequent 3-D image reconstruction of the posterior pole. In the study described herein, we prospectively examined the macular area in a group of consecutive eyes with CSC using a $1-\mu \mathrm{m}$ wavelength swept-source OCT to highlight the prevalence, morphology and 3-D tomographic features of focal choroidal excavation, and the possible correlation between focal choroidal excavation and the pathogenesis of CSC. 


\section{METHODS}

The Ethics Committee at Kyoto University Graduate School of Medicine approved this prospective study, which was conducted in accordance with the tenets of the Declaration of Helsinki. Written informed consent was obtained from each subject before any study procedures or examinations were performed.

We prospectively examined 116 eyes from 99 consecutive CSC patients who presented to the macula clinic at Kyoto University Hospital between June 2010 and January 2013. All study subjects were Japanese. All study participants underwent a comprehensive ocular examination, including autorefractometry, best-corrected visual acuity measurement in a Landolt chart, slit-lamp biomicroscopy, intraocular pressure measurement, fundus photography (TRC-NW8F, Topcon Corp., Tokyo, Japan), and 3-D swept-source OCT imaging with a prototype system (Topcon Corp.). Simultaneous FA and ICGA, by using the Spectralis HRA+OCT (Heidelberg Engineering, Heidelberg, Germany), were performed in most eyes as indicated by the clinical course of the CSC.

\section{Diagnosis and Classification of Central Serous Chorioretinopathy} CSC was diagnosed based on medical history, serous retinal detachment seen on the fundus examination and OCT, and angiographic leakage(s) at the level of retinal pigment epithelium (RPE) in FA. Patients with other causes of fluorescein leakage (e.g., age-related macular degeneration, polypoidal choroidal vasculopathy, idiopathic choroidal neovascularization, other secondary neovascular maculopathy) or other causes of serous retinal detachment unrelated to CSC (e.g., intraocular inflammation, posterior segment tumors) were excluded from the study.

All CSC cases were classified by FA results into 3 types: ${ }^{28}$ classic CSC, chronic CSC, and multifocal posterior pigment epitheliopathy. ${ }^{29,}{ }^{30}$ Eyes showing only one or few specific angiographic leakage points at the level of RPE were classified as classic CSC. Eyes with broad areas of granular hyperfluorescence on FA associated with indistinct areas of leakage were classified as chronic CSC. Multifocal posterior pigment epitheliopathy was defined as multiple massive leakages from the choroid into the subretinal space. ${ }^{29}$

All eyes were divided into active and resolved eyes on the basis of the presence of serous retinal detachment at the time of swept-source OCT examination. Active CSC was defined as the presence of a serous retinal detachment and resolved CSC was defined as the absence of a serous retinal detachment. Eyes with resolved CSC that were included in this study had active disease either at the initial clinic visit or at other follow-up 
visits, but before swept-source OCT examinations.

\section{Swept-Source Optical Coherence Tomography and Scan Protocols}

The prototype swept-source OCT used in the current study has been previously described in details. ${ }^{31-33}$ Briefly, this swept-source OCT uses a light source of a wavelength-sweeping laser centered at $1050 \mathrm{~nm}$ with a tuning range of $100 \mathrm{~nm}$. This system has a scanning speed of 100,000 A-scans per second and a scan window depth of $2.6 \mathrm{~mm}$. The axial and transverse resolutions are $8 \mu \mathrm{m}$ and $20 \mu \mathrm{m}$ in tissue, respectively. The optical power incident on the cornea with the current swept-source laser system is less than $1 \mathrm{~mW}$, which is below the safety requirements for this laser class by the American National Standards Institute.

Swept-source OCT examinations were performed by trained examiners after pupil dilation. In each subject, multi-averaged horizontal and vertical scans of $12 \mathrm{~mm}$ were obtained. Fifty single images, where each image consisted of 1,024 A-scans, were registered and averaged by software to create a multi-averaged single image. A raster scan protocol of 512 (horizontal) $\times 128$ (vertical) A-scans per dataset was acquired in 0.8 seconds to create $3-\mathrm{D}$ datasets (total, 65,536 axial scans/volume). Each raster scan consisted of $128 \mathrm{~B}$-scans and covered an area of $6 \mathrm{~mm} \times 6 \mathrm{~mm}$, centered on the fovea (Supplemental Movie 1). The centration of the scan was achieved with internal fixation target and confirmed by a built-in camera within the swept-source OCT system. Owing to the high speed and the invisible scanning light wavelength, eye movements during the 3-D image acquisition were minimal. To decrease speckle noise, each image was de-noised by the weighted moving average of 3 consecutive original B-scans.

\section{Focal Choroidal Excavation}

Diagnosis of focal choroidal excavation was based on the presence of an excavated area into the choroid, along the RPE/Bruch's membrane complex line on OCT scans, without any history of prior trauma, infection, or inflammatory episode.

Focal choroidal excavation was classified by OCT as conforming; if photoreceptor tips were attached to the apical surface of the RPE, or as non-conforming; if there was a separation between the photoreceptor tips and the RPE. ${ }^{20}$ In terms of location, focal choroidal excavations were classified as foveal, if the foveal center was located within the excavation, or as extra-foveal, if the foveal center was located outside the excavation.

In eyes with focal choroidal excavations, 3-D topographical images were reconstructed from the OCT scans, by segmentation of the line of the outer surface of RPE, to highlight the shape of the excavation. In each B-scan, the outer surface of RPE 
line was automatically determined by the software and manual corrections were done, as necessary, using the built-in segmentation-modifying tool. The excavation depth and width were measured manually from the 3-D dataset in the scan that showed the greatest dimensions. En-face OCT scans along the z-axis (C-scans) were obtained from the 3-D data set and the scan showing the excavation at the level of the RPE plane was selected. The excavation boundary in the en-face image was marked by a hyperreflective band, representing the RPE.

\section{Tomographic and Angiographic Features}

The tomographic and angiographic features at the area of excavation were examined. Additionally, the correlation between the locations of the excavation and the leakage areas in FA, and areas of choroidal hyperpermeability and punctate hyperfluorescent spots in ICGA were analyzed. Choroidal hyperpermeability was defined as cloud-like areas of hyperfluorescence with blurred margins within the choroid in the mid to late phase of ICGA. ${ }^{4}$ Punctate hyperfluorescent spots were defined as a cluster of tiny hyperfluorescent spots in the late phase of ICGA within the macular area. ${ }^{2}$

The retinal and choroidal thicknesses at the center of the fovea were manually measured with a built-in caliber tool within the software. Retinal thickness was defined as the distance between the vitreoretinal interface and the outer border of the RPE and choroidal thickness was defined as the distance between the outer border of the RPE and the chorioscleral interface. When the focal choroidal excavation was located within the foveal center, choroidal thickness measurements were made in nearby choroid that was not affected by the excavation.

\section{Statistical Analyses}

All values are expressed as mean \pm standard deviation. The measured visual acuity was converted to the logarithm of the minimum angle of resolution (logMAR) for statistical analyses. Unpaired t-tests were used to compare numerical variable means and Fisher's exact tests were used to compare the distribution of categorical variables. Statistical significance was defined as a $P<.05$.

\section{RESULTS}

Of the 116 eyes examined (99 consecutive patients with CSC), 3-D swept-source OCT examination detected focal choroidal excavation in 9 eyes $(7.8 \%)$ from 8 patients. The mean age of eyes with focal choroidal excavation was $54.9 \pm 13.6$ years (range: 35 to 76 
years) and all 9 eyes were myopic, with a mean spherical equivalent of $-4.42 \pm 2.92$ diopters. Mean foveal retinal thickness was $194.4 \pm 50.6 \mu \mathrm{m}$ (range: 125 to $311 \mu \mathrm{m}$ ) and mean foveal choroidal thickness was $301.3 \pm 60.9 \mu \mathrm{m}$ (range: 192 to $376 \mu \mathrm{m}$ ). Table 1 shows the patient demographics and ocular tomographic features of eyes with focal choroidal excavations. Microperimetry data was available in 3 eyes, all of which had decreased retinal sensitivity at the excavation area.

\section{Tomographic Features of Eyes with Focal Choroidal Excavation}

The 3-D scanning protocol and en-face OCT scans allowed us to detect focal choroidal excavations in the macular area and to clearly visualize their morphology. The focal choroidal excavation had a foveal location in 3 eyes and an extrafoveal location in the remaining 6 eyes. In two eyes, extrafoveal excavations were detected with the 3-D scanning protocol, but not with the line scanning protocol (Fig.1). In addition, the 3-D reconstructed images of the macular area showed that focal choroidal excavations can vary in shape, ranging between small dimple-like excavations (Fig. 2) to broad irregular excavations (Fig. 3). En-face images at the level of the RPE showed whether the excavation was filled with outer retinal tissue (conforming excavation) or if the excavation was optically empty, possibly filled with subretinal fluid (non-conforming excavation).

The mean depth of the excavation in the 9 eyes was $69.2 \pm 20.7 \mu \mathrm{m}$ (range: $36-95$ $\mu \mathrm{m}$ ) and mean excavation width was $727.0 \pm 318.0 \mu \mathrm{m}$ (range: $337-1,237 \mu \mathrm{m}$ ). In all 9 eyes, the inner retinal layers appeared physiologically normal and even when the focal choroidal excavation was located subfoveally; the foveal contour remained nearly well-preserved. At the excavation, the line of the external limiting membrane was preserved in $8(88.9 \%)$ eyes and disrupted in one (11.1\%) eye. The line of the junction between the inner and outer segments of the photoreceptors remained continuous in 3 (33.3\%) eyes, was partially disrupted in 5 (55.6\%) eyes, and was completely disrupted in $1(11.1 \%)$ eye. The RPE line was intact in all 9 eyes, despite some thinning or attenuation.

Swept-source OCT allowed visualization of choroidal structures. Multi-averaged scans often showed an inner choroidal layer with medium-diameter blood vessels and an outermost choroidal layer with larger-diameter blood vessels. In 5 eyes with focal choroidal excavation, unusual choroidal tissue without large vessels was detected beneath the excavation, bridging between the bottom of the excavation and the outer choroidal boundary (Fig. 3). In addition, the suprachoroidal space below the focal excavation was observed in 3 of these 5 eyes, as if the outer choroidal boundary was pulled inward by the bridging tissue (Fig. 4). The chorioscleral interface was 
physiologically smooth, with no ectasia, in all 9 eyes.

The mean follow up duration of eyes with focal choroidal excavation by OCT examination was $42.8 \pm 31.4$ months (range: 4 to 78 months). In all 9 eyes, the excavations were located within the serous retinal detachment during the active stage (non-conforming excavation). During the follow up period, 3 eyes showed resolution of the serous retinal detachment and the photoreceptors tips reattached to the RPE, thus, converting the focal choroidal excavation from the non-conforming to the conforming type. In one eye, the serous retinal detachment completely resolved, but the photoreceptor tips remained separated from the RPE at the area of excavation. In the 5 eyes that were followed for more than 4 years with OCT, no remarkable changes in excavation size or shape were detected. Additionally, no eye developed new focal choroidal excavation and no eye showed disappearance of the excavation in the macular area during the follow up.

\section{Fundus and Angiographic Features in Eyes with Central Serous Chorioretinopathy and Focal Choroidal Excavation}

All eyes with focal choroidal excavations showed changes in color fundus photographs at the area of the excavation (Table 2). The focal choroidal excavations appeared either as a yellowish lesion with indistinct margins ( $\mathrm{n}=3$ eyes) or as a pigmentary disturbance, which often extend beyond the area of excavation ( $n=6$ eyes). FA was performed in all 9 eyes, 3 (33.3\%) of which showed classic CSC and the remaining $6(66.7 \%)$ eyes had chronic type leakage. In 8 (88.9\%) of the 9 eyes, the focal excavation was located within the area of fluorescein leakage, as either a focal leak (classic CSC) or within the area of granular hyperfluorescence (chronic CSC). In the remaining eye, the focal choroidal excavation was located just adjacent to the focal leakage area in FA. ICGA was performed in 7 eyes, all of which showed choroidal hyperpermeability and punctate hyperfluorescent spots. In all 7 eyes, the focal choroidal excavations were seen within the area of choroidal hyperpermeability (Fig. 3).

\section{Comparison between Central Serous Chorioretinopathy with and without Focal Choroidal Excavation}

Table 3 shows data comparisons of CSC eyes with and without focal choroidal excavation. There were no significant differences in age, visual acuity, or gender distribution between the two groups. However, eyes with focal choroidal excavations were more myopic (-4.42 \pm 2.92 diopters) than eyes without the excavations $(-0.27 \pm 1.80$ diopters, $P=.001)$. The foveal retinal thickness in eyes without focal choroidal excavation $(270.8 \pm 108.7 \mu \mathrm{m})$ was significantly greater than eyes with the excavations 
(194.4 $\pm 50.6 \mu \mathrm{m}, P=.040)$. The subfoveal choroid was significantly thinner in eyes with focal choroidal excavations $(301.3 \pm 60.9 \mu \mathrm{m})$ than in eyes without the excavations (376.6 $\pm 104.8 \mu \mathrm{m}, P=.036)$. The foveal choroidal thickness in the fellow eyes of patients with focal choroidal excavation was $269.4 \pm 97.0 \mu \mathrm{m}$. There was no statistical difference in the foveal choroidal thickness between both eyes $(P=.418)$.

\section{DISCUSSION}

The current study showed that focal choroidal excavations are seen in $7.8 \%$ of eyes with CSC. Focal choroidal excavation could be suspected by careful ophthalmoscopic examination appearing as an indistinct yellowish lesion or as pigmentary mottling, but only confirmed by OCT examination. 3-D reconstructed OCT images showed that the excavations often have irregular shapes and dip variably into the choroid, and the en-face scans highlighted the actual transverse shape of the excavation. Additionally, 3-D OCT scanning of the macula helps to detect tiny focal choroidal excavations that could be easily missed in a routine OCT examination. Because all eyes with focal choroidal excavation had a normal appearing inner scleral surface with no out-pouching, we believe that focal choroidal excavation is essentially a focal choroidal abnormality.

Recent OCT image analysis revealed choroidal thickening in eyes with CSC, especially in the active phase. ${ }^{12,13}$ In the current study, subfoveal choroidal thickness in eyes with focal choroidal excavation (301.3 $\pm 60.9 \mu \mathrm{m}$; mean age, $54.9 \pm 13.6$ years) was greater than that observed in normal Japanese subjects of the same age group (240.2 \pm $66.8 \mu \mathrm{m}$; mean age, $54.1 \pm 3.1$ years). ${ }^{34}$ In addition, Jirarattanasopa and associates ${ }^{11}$ reported a local choroidal thickening in areas of choroidal vascular hyperpermeability and in punctate hyperfluorescent spots. In our patients, 3-D OCT and ICGA images showed that focal choroidal excavations were located within these areas of choroidal abnormalities and that focal choroidal excavation may be a feature associated with choroid thickening.

The foveal retinal thickness in CSC eyes without focal choroidal excavation $(270.8$ $\pm 108.7 \mu \mathrm{m})$ was significantly greater than that in eyes with the excavations $(194.4 \pm 50.6$ $\mu \mathrm{m})$. The reason for this difference is uncertain. In our patients with CSC, the eyes without excavations have more percentage of active CSC with serous retinal detachment $(64.5 \%)$, as compared to eyes with excavations (44.4\%) at the time of swept source OCT examination. The percentage of active CSC may be involved in this difference in the foveal retinal thickness. Additionally, the difference in the height of the serous 
detachment in both groups may have contributed to this difference.

Theoretically, there are two possible directional forces that can lead to the formation of the focal choroidal excavation, either forces pushing the retinal parenchyma and RPE into the choroid or pulling forces causing traction on the RPE toward the choroid. In 5 eyes with focal choroidal excavations, unusual choroidal tissue, hyperreflective on OCT and devoid of large choroidal vessels, was detected beneath the excavation bridging the bottom of the focal excavation and the outer choroidal boundary. Additionally, in 3 of these 5 eyes, the suprachoroidal space was seen below the focal choroidal excavation, as if the outer boundary of the choroid was pulled inward by this bridging tissue. The nature of this unusual tissue is unknown, however, it may represent focal scarring in the choroidal connective tissue and subsequent contraction of such scar results in focal retraction of the RPE, leading to the formation of the focal excavation.

In addition, the shape of most excavations was irregular and often pointed externally, rather than being a smooth out-pouching. The presence of an outward traction on the RPE would explain this shape. Alternatively, such choroidal scarring can causes a contractive force on the RPE when the choroid is thickened due to CSC during the active stage of the disease and such abnormal tractional force may lead to focal choroidal excavation development. However, this unusual choroidal tissue was detected in only 5 of 9 eyes with focal excavation and, therefore, other pathogenic mechanisms may be involved in the formation of the focal choroidal excavation.

It is unknown whether focal choroidal excavation is a congenital choroidal malformation or an acquired insult. Previously, Margolis and associates ${ }^{20}$ and Abe and associates $^{22}$ suggested that focal choroidal excavation may result from a pre-existing congenital malformation. Such lesion can cause visual disturbances if secondary changes occur later in lifetime. In our patients, no patient developed new focal excavation during the course of the disease and no eyes showed the resolution of the focal excavation during the follow up. In addition, in 5 eyes with follow up of more than 4 years, the focal choroidal excavation remained almost stable without any remarkable change in size or shape during the OCT follow up period.

In the current study, all focal choroidal excavations were located within or adjacent to areas of fluorescein leakage and in all 7 eyes examined with ICGA, the focal excavations were located within areas of choroidal hyperpermeability. One can speculate that focal choroidal excavation has a possible role in the pathogenesis of CSC; however, because the prevalence of focal choroidal excavation was low (7.8\%) in CSC, the role would be limited only to some eyes. 
Although eyes with CSC are often emmetropic or slightly hyperopic, all 9 eyes in our study with focal choroidal excavation associated with CSC were myopic. The reason for this observation is unknown. However, this observation is consistent with previous case reports. $^{18,19,21-23}$ In the current study, subfoveal choroidal thickness in eyes with focal choroidal excavation was greater than in normal Japanese subjects of the same age group. ${ }^{34}$ However, foveal choroidal thickness in CSC eyes with focal choroidal excavation has been shown to be significantly thinner than in with CSC eyes without the excavation. ${ }^{11,13}$ One possible explanation is the myopic trend seen in eyes with CSC and focal choroidal excavations, and previous studies have shown that choroidal thickness is negatively correlated with refractive error. ${ }^{35,36}$

Focal choroidal excavation was initially reported as an incidental finding in patients complaining of metamorphopsia. ${ }^{21-23}$ It was later shown that focal choroidal excavations are associated with vision threatening diseases, including choroidal neovascularization ${ }^{18}$ and polypoidal choroidal vasculopathy ${ }^{19}$ and these previous reports showed that focal choroidal excavations were located within the areas of pathology. In the current study, focal choroidal excavations were also located within areas of angiographic leakage and choroidal hyperpermeability in eye with CSC. The pathogenesis of these aforementioned diseases is thought to be choroidal in nature and focal choroidal excavations are thought of primarily as a choroidal abnormality. However, it is still unclear whether focal choroidal excavation is a platform for the development of such complications.

The current study had some limitations. First, the number of eyes with focal choroidal excavation was small. Second, all subjects were Asian and it is possible that the prevalence of focal choroidal excavation is different in other ethnicities. Third, little is known about the natural progression of focal choroidal excavation and it remains unclear whether focal choroidal excavation is a congenital or acquired abnormality. So far, the histopathologic nature of the focal choroidal excavation is not known and further speculation into its pathogenic mechanisms is difficult. In addition, the lack of normal control eyes without CSC does not prove a true association between CSC and focal choroidal excavation.

In conclusion, focal choroidal excavation is not very rare in eyes with CSC, with a prevalence of approximately $7.8 \%$. Although the focal choroidal excavation might be partly related to the pathogenesis of CSC, its role is likely to be limited to some eyes. The diagnosis of focal choroidal excavation can only be made with OCT, however, it could be suspected by careful fundus examination. The 3-D reconstructed images revealed the shape of the focal excavations and provided more insight into their morphology. However, the clinical importance of focal choroidal excavation is still unclear. 


\section{ACKNOWLEDGMENTS}

A. Funding/Support: This study was supported, in part, by the Japan Society for the Promotion of Science (JSPS), Tokyo, Japan (Grant-in-Aid for Scientific Research, no. 21592256), and by the Japan National Society for the Prevention of Blindness, Tokyo, Japan.

B. Financial Disclosure: N. Yoshimura, Topcon Corporation (Financial Support), Nidek (Financial Support, Consultant), Canon (Financial Support); A. Tsujikawa, Pfizer (Grant support).

C. Contributions of Authors: Conception and design of the study (AAE, A Tsujikawa, NY); analysis and interpretation (AAE, A Tsujikawa, KY, AO, SO, IN, MM, YA-K, NU-A, SA, SY, A Takahashi); writing of the article (AAE, A Tsujikawa); critical revision of the article (KY, AO, SO, IN, MM, YA-K, NU-A, SA, SY, A Takahashi, NY); final approval of the article (AAE, A Tsujikawa, SO, KY, AO, IN, YA-K, MM, NY); data collection (AAE, KY, AO, SO, IN, MM, YA-K, NU-A, SA, SY, A Takahashi).

D. Other Acknowledgments: none.

\section{REFERENCES}

1. lida T, Kishi S, Hagimura N, Shimizu K. Persistent and bilateral choroidal vascular abnormalities in central serous chorioretinopathy. Retina 1999;19(6):508-512.

2. Tsujikawa A, Ojima Y, Yamashiro K, et al. Punctate hyperfluorescent spots associated with central serous chorioretinopathy as seen on indocyanine green angiography. Retina 2010;30(5):801-809.

3. Kitaya N, Nagaoka T, Hikichi T, et al. Features of abnormal choroidal circulation in central serous chorioretinopathy. Br J Ophthalmol 2003;87(6):709-712.

4. Guyer DR, Yannuzzi LA, Slakter JS, Sorenson JA, Ho A, Orlock D. Digital indocyanine green videoangiography of central serous chorioretinopathy. Arch Ophthalmol 1994;112(8):1057-1062.

5. Kuroda S, Ikuno Y, Yasuno Y, et al. Choroidal thickness in central serous chorioretinopathy. Retina 2013;33(2):302-308.

6. Prunte C, Flammer J. Choroidal capillary and venous congestion in central serous chorioretinopathy. Am J Ophthalmol 1996;121(1):26-34. 
7. Prunte C. Indocyanine green angiographic findings in central serous chorioretinopathy. Int Ophthalmol 1995;19(2):77-82.

8. Menchini U, Virgili G, Lanzetta P, Ferrari E. Indocyanine green angiography in central serous chorioretinopathy. ICG angiography in CSC. Int Ophthalmol 1997;21(2):57-69.

9. Scheider A, Nasemann JE, Lund OE. Fluorescein and indocyanine green angiographies of central serous choroidopathy by scanning laser ophthalmoscopy. Am J Ophthalmol 1993;115(1):50-56.

10.Spaide RF, Koizumi H, Pozzoni MC. Enhanced depth imaging spectral-domain optical coherence tomography. Am J Ophthalmol 2008;146(4):496-500.

11.Jirarattanasopa P, Ooto S, Tsujikawa A, et al. Assessment of macular choroidal thickness by optical coherence tomography and angiographic changes in central serous chorioretinopathy. Ophthalmology 2012;119(8):1666-1678.

12.Imamura Y, Fujiwara T, Margolis R, Spaide RF. Enhanced depth imaging optical coherence tomography of the choroid in central serous chorioretinopathy. Retina 2009;29(10):1469-1473.

13. Maruko I, lida T, Sugano Y, Ojima A, Ogasawara M, Spaide RF. Subfoveal choroidal thickness after treatment of central serous chorioretinopathy. Ophthalmology 2010;117(9):1792-1799.

14.Kim YT, Kang SW, Bai KH. Choroidal thickness in both eyes of patients with unilaterally active central serous chorioretinopathy. Eye (Lond) $2011 ; 25(12): 1635-1640$.

15.Pryds A, Larsen M. Choroidal thickness following extrafoveal photodynamic treatment with verteporfin in patients with central serous chorioretinopathy. Acta Ophthalmol 2012;90(8):738-743.

16.Nicholson B, Noble J, Forooghian F, Meyerle C. Central serous chorioretinopathy: update on pathophysiology and treatment. Surv Ophthalmol 2013;58(2):103-126.

17. Uetani R, Ito Y, Oiwa K, Ishikawa K, Terasaki H. Half-dose vs one-third-dose photodynamic therapy for chronic central serous chorioretinopathy. Eye (Lond) 2012;26(5):640-649.

18.Katome T, Mitamura Y, Hotta F, Niki M, Naito T. Two cases of focal choroidal excavation detected by spectral-domain optical coherence tomography. Case Report Ophthalmol 2012;3(1):96-103.

19.Kobayashi W, Abe T, Tamai H, Nakazawa T. Choroidal excavation with polypoidal choroidal vasculopathy: a case report. Clin Ophthalmol 2012;6:1373-1376.

20.Margolis R, Mukkamala SK, Jampol LM, et al. The expanded spectrum of focal choroidal excavation. Arch Ophthalmol 2011;129(10):1320-1325. 
21.Wakabayashi Y, Nishimura A, Higashide T, ljiri S, Sugiyama K. Unilateral choroidal excavation in the macula detected by spectral-domain optical coherence tomography. Acta Ophthalmol 2010;88(3):e87-91.

22. Abe S, Yamamoto T, Kirii E, Yamashita H. Cup-Shaped Choroidal Excavation Detected By Optical Coherence Tomography: A Case Report. Retinal Cases and Brief Reports 2010;4(4):373-376.

23.Jampol LM, Shankle J, Schroeder R, Tornambe P, Spaide RF, Hee MR. Diagnostic and therapeutic challenges. Retina 2006;26(9):1072-1076.

24.Chen JC, Gupta RR. A case of multiple focal choroidal excavations. Can J Ophthalmol 2012;47(6):e56-58.

25.Motaghiannezam R, Schwartz D, Fraser SE. In vivo human choroidal vascular pattern visualization using high speed swept source optical coherence tomography at 1060 nm. Invest Ophthalmol Vis Sci 2012;53(4):2337-2346.

26. Ellabban AA, Tsujikawa A, Matsumoto A, et al. Macular choroidal thickness measured by swept source optical coherence tomography in eyes with inferior posterior staphyloma. Invest Ophthalmol Vis Sci 2012;53(12):7735-7745.

27.Ohno-Matsui K, Akiba M, Moriyama M, Ishibashi T, Tokoro T, Spaide RF. Imaging retrobulbar subarachnoid space around optic nerve by swept-source optical coherence tomography in eyes with pathologic myopia. Invest Ophthalmol Vis Sci 2011;52(13):9644-9650.

28.Klais C, Ober M, Ciardella A, Yannuzzi L. Central serous chorioretinopathy, 4th ed. In: Rayan SJ e-i-c, Hinton DR, Schachat AP, Wilkinson CP, editor. Retina. Philadelphia, PA: Elsevier/Mosby, 2006:1135-1161.

29. Uyama M, Matsunaga H, Matsubara T, Fukushima I, Takahashi K, Nishimura T. Indocyanine green angiography and pathophysiology of multifocal posterior pigment epitheliopathy. Retina 1999;19(1):12-21.

30.Gass JD. Bullous retinal detachment. An unusual manifestation of idiopathic central serous choroidopathy. Am J Ophthalmol 1973;75(5):810-821.

31.Ellabban AA, Tsujikawa A, Matsumoto A, et al. Three-Dimensional tomographic features of dome-shaped macula by swept-source optical coherence tomography. $A m$ J Ophthalmol 2012;155(2):320-328.e322.

32. Hirata M, Tsujikawa A, Matsumoto A, et al. Macular choroidal thickness and volume in normal subjects measured by swept-source optical coherence tomography. Invest Ophthalmol Vis Sci 2011;52(8):4971-4978.

33. Ellabban AA, Tsujikawa A, Matsumoto A, et al. Macular choroidal thickness and volume in eyes with angioid streaks measured by swept source optical coherence 
tomography. Am J Ophthalmol 2012;153(6):1133-1143.

34.Fujiwara A, Shiragami C, Shirakata Y, Manabe S, Izumibata S, Shiraga F. Enhanced depth imaging spectral-domain optical coherence tomography of subfoveal choroidal thickness in normal Japanese eyes. Jpn J Ophthalmol 2012;56(3):230-235.

35. Nishida Y, Fujiwara T, Imamura Y, Lima LH, Kurosaka D, Spaide RF. Choroidal thickness and visual acuity in highly myopic eyes. Retina 2012;32(7):1229-1236. 36.Flores-Moreno I, Lugo F, Duker JS, Ruiz-Moreno JM. The relationship between axial length and choroidal thickness in eyes with high myopia. Am J Ophthalmol 2013;155(2):314-319 e311.

\section{FIGURE CAPTIONS}

Figure 1. Extrafoveal focal choroidal excavation examined with swept-source optical coherence tomography (OCT) in 4 eyes with central serous chorioretinopathy. (Left column) Fundus photographs of 4 eyes with focal choroidal excavations. (Second column) Magnified fundus images (2x) of the dashed green square in the left column. Focal excavations were detected in fundus photographs as either indistinct yellowish lesions or pigmentary mottling (white arrows). (Third column) Reconstructed three-dimensional (3-D) $6 \mathrm{~mm} \times 6 \mathrm{~mm}$ images (dashed green squares in fundus photographs) of the retinal pigment epithelium showing the shape of the excavations. (Right column) The 3-D volumetric data set of a scan positioned over the focal choroidal excavation (dashed green lines in fundus photographs). The focal choroidal excavations in the first and second rows were extrafoveal and were detectable only on 3-D scans. The white asterisks indicate the foveal center and the black arrows indicate OCT scan direction.

Figure 2. A small dimple-like focal choroidal excavation seen in an eye with central serous chorioretinopathy (CSC). (Top row, left) Fundus photograph of the right eye of a 60-year-old female with resolved CSC (Patient 3). Pigmentary mottling at the area corresponding to the focal choroidal excavation can be seen (white arrowhead). The dashed green square outlines the area $\left(6 \times 6 \mathrm{~mm}^{2}\right)$ scanned by the swept-source optical coherence tomography (OCT). (Top, middle) Reconstructed three-dimensional (3-D) image by segmentation of the retinal pigment epithelium (RPE) shows the 3-D shape of the excavation. (Top, right) En-face image at the level of RPE shows the transverse shape of the excavation. (Second row, left) Horizontal and vertical (bottom, left) OCT 
sections through the focal choroidal excavation. (Second row, right) Magnified image in the area outlined by the dashed red square. The external limiting membrane was preserved, the photoreceptor inner/outer segment junction line was partially disrupted, and the RPE was almost preserved. The chorioscleral interface appears normal, without any ectasia. (Bottom, right) Horizontal OCT section at the fovea (upper) shows a non-conforming focal choroidal excavation at the time of active CSC with serous retinal detachment. With the resolution of the serous retinal detachment (lower), the retina reattached to RPE surface. No remarkable changes in the focal choroidal excavation size or shape occurred during the follow up. The white asterisk indicates the foveal center and the white arrows indicate the direction of the OCT scan.

Figure 3. Broad irregular focal choroidal excavation in an eye with central serous chorioretinopathy (CSC). (Top, left) Fundus photograph of the left eye of a 35-year-old man (Patient 4). Best-corrected visual acuity was 1.5 (Snellen: 20/13). Fundus photograph showing pigmentary mottling in the area corresponding to the focal choroidal excavation (white arrowhead). The dashed green square outlines the area $\left(6 \times 6 \mathrm{~mm}^{2}\right)$ scanned by the swept-source optical coherence tomography (OCT). (Top, second) Fundus autofluorescence image obtained at the active phase of CSC shows mixed areas of hyper- and hypo-autofluorescence in the area of focal choroidal excavation. (Top, third) Fluorescein angiography obtained at the active phase of CSC shows chronic pattern of leakages. (Top row, fourth) Late phase indocyanine green angiography obtained at the active phase of CSC showing multiple areas of choroidal hyperpermeability and punctate hyperfluorescent spots. The focal choroidal excavation was located within one of the areas of choroidal hyperpermeability and punctate hyperfluorescent spots. (Top, right) Interpolated color map of microperimetry data shows decreased retinal sensitivity at the area of excavation. (Second row) Horizontal (left) and vertical (right) OCT sections at the fovea showing a conforming focal choroidal excavation with a broad, irregular shape. Beneath the focal choroidal excavation, no large choroidal vessels are visible. Unusual choroidal tissue (yellow arrow) is seen bridging between the bottom of the focal excavation and the outer choroidal boundary. (Bottom, left) Reconstructed image by the segmentation of the retinal pigment epithelium (RPE) shows the three-dimensional shape of the excavation. (Bottom, middle) En-face image at the level of the RPE shows the transverse shape of the excavation. (Bottom, right) Consecutive horizontal (left) and vertical (right) OCT scans obtained at follow-up visits, the focal choroidal excavation changed from non-conforming to conforming type with the resolution of the serous detachment. There were no 
remarkable changes in the dimensions or the shape of the excavation over the follow up period of 6 years. The white arrows indicate the direction of the OCT scan.

Figure 4. Suprachoroidal space under the focal choroidal excavation in an eye with central serous chorioretinopathy (CSC). (Top, left) Fundus photograph of the left eye of a 76-year-old man (Patient 1). Best-corrected visual acuity was 1.2 (Snellen: 20/16). (Top, second). Magnified fundus image in the area outlined by the dashed green square shows an indistinct yellowish lesion at the location of the irregular-shaped excavation (white arrowhead). (Top, third) Reconstructed image by the segmentation of the retinal pigment epithelium (RPE) shows the three-dimensional (3-D) shape of the excavation. (Top, right) En-face image at the level of the RPE shows the transverse shape of the excavation. (Second row, left) Horizontal OCT section through the fovea shows a serous retinal detachment under the fovea. (Bottom, left) Vertical OCT section through the fovea shows a non-conforming focal choroidal excavation. (Bottom, right) A 3-D volumetric data set with scan positioned at the area involving the focal choroidal excavation (along the dashed green line in fundus photograph). Beneath the focal choroidal excavation, unusual choroidal tissue, devoid of large vessels, is seen bridging the bottom of the excavation and the outer choroidal boundary (yellow arrows). The suprachoroidal space (red arrows) is seen under the focal choroidal excavation and the chorioscleral interface appears normal, with no ectasia. The white asterisk indicates the foveal center. 
FOCAL CHOROIDAL EXCAVATION IN CENTRAL SEROUS CHORIORETINOPATHY. Ellabban AA, et al.

Table 1. Clinical Characteristics of Eyes with Focal Choroidal Excavation Associated with Central Serous Chorioretinopathy

\begin{tabular}{|c|c|c|c|c|c|c|c|c|c|c|c|c|c|c|}
\hline \multirow[b]{5}{*}{ Patient } & \multirow{5}{*}{$\begin{array}{c}\text { Age } \\
\text { (years) }\end{array}$} & \multirow[b]{5}{*}{ Sex } & \multirow{3}{*}{\multicolumn{3}{|c|}{$\begin{array}{l}\text { Refractive } \\
\text { Error } \\
\text { Spherical }\end{array}$}} & \multirow{5}{*}{$\begin{array}{c}\text { Excavation } \\
\text { Type }\end{array}$} & \multirow{5}{*}{$\begin{array}{l}\text { CSC } \\
\text { Type }\end{array}$} & \multirow{5}{*}{$\begin{array}{c}\text { CSC } \\
\text { Activity }\end{array}$} & \multirow{5}{*}{$\begin{array}{l}\text { FRT } \\
(\mu \mathrm{m})\end{array}$} & \multirow{5}{*}{$\begin{array}{l}\text { FChT } \\
(\mu \mathrm{m})\end{array}$} & \multirow{5}{*}{$\begin{array}{c}\text { Exca- } \\
\text { vation } \\
\text { Depth } \\
(\mu \mathrm{m})\end{array}$} & \multirow{3}{*}{$\begin{array}{l}\text { Exca- } \\
\text { vation }\end{array}$} & \multirow{5}{*}{\multicolumn{2}{|c|}{$\begin{array}{l}\text { Duration of } \\
\text { Follow-up with } \\
\text { OCT } \\
\text { examination } \\
\text { tmonths) }\end{array}$}} \\
\hline & & & & & & & & & & & & & & \\
\hline & & & & & & & & & & & & & & \\
\hline & & & Visual & Equivalent & & & & & & & & Width & & \\
\hline & & & Acuity $^{b}$ & (diopters) & Location & & & & & & & $(\mu \mathrm{m})$ & & \\
\hline 1 & 76 & M & 1.2 & -0.50 & Extrafoveal & Non-conforming & Chronic & Active & 213 & 331 & 95 & 725 & 0 & 52 \\
\hline 2 & 52 & $\mathrm{~F}$ & 1.2 & -10.50 & Foveal & Non-conforming & Classic & Active & 311 & 267 & 50 & 902 & rfPDT & 12 \\
\hline 3 & 60 & $\mathrm{~F}$ & 1.2 & -2.75 & Extrafoveal & $\begin{array}{l}\text { Non-Conforming } \\
=>\text { Conforming }^{c}\end{array}$ & Classic & Resolved & 185 & 228 & 87 & 623 & 0 & 58 \\
\hline \multirow[t]{2}{*}{$4^{a}$} & 35 & M & 1.2 & -3.75 & Extrafoveal & Non-conforming & Chronic & Active & 190 & 376 & 54 & 580 & 0 & 78 \\
\hline & 35 & M & 1.5 & -5.75 & Foveal & $\begin{array}{l}\text { Non-Conforming } \\
=>\text { Conforming }\end{array}$ & Chronic & Resolved & 197 & 328 & 91 & 1237 & 0 & 78 \\
\hline 5 & 50 & M & 1.5 & -5.50 & Extrafoveal & Non-conforming & Chronic & Active & 195 & 359 & 36 & 414 & 0 & 24 \\
\hline 6 & 62 & $\mathrm{~F}$ & 1.2 & -5.75 & Extrafoveal & Non-conforming & Classic & Resolved & 173 & 192 & 69 & 337 & 0 & 73 \\
\hline 7 & 58 & M & 1.0 & -1.75 & Extrafoveal & Non-conforming & Chronic & Active & 161 & 328 & 59 & 547 & 0 & 4 \\
\hline 8 & 66 & $M$ & 0.1 & -3.50 & Foveal & $\begin{array}{l}\text { Non-Conforming } \\
=>\text { Conforming }\end{array}$ & Chronic & Resolved & 125 & 303 & 82 & 1178 & rfPDT & 6 \\
\hline
\end{tabular}

CSC = central serous chorioretinopathy; FRT = foveal retinal thickness; FChT = foveal choroidal thickness; rfPDT = reduced fluence photodynamic therapy; OCT = optical coherence tomography. ${ }^{a}$ Patient 4 has bilateral focal choroidal excavation. ${ }^{b}$ Landolt visual acuity. ${ }^{\circ}$ The focal choroidal excavation changed from non-conforming to conforming type upon the resolution of serous detachment in 3 eyes. 
FOCAL CHOROIDAL EXCAVATION IN CENTRAL SEROUS CHORIORETINOPATHY. Ellabban AA, et al.

Table 2. Angiographic Characteristic of Eyes with Focal Choroidal Excavation Associated with Central Serous Chorioretinopathy

\begin{tabular}{|c|c|c|c|c|c|c|}
\hline Patient & $\begin{array}{c}\text { Color } \\
\text { Photography }\end{array}$ & $\begin{array}{c}\text { Fundus } \\
\text { Autofluorescence }\end{array}$ & $\begin{array}{c}\text { Correlation between Focal } \\
\text { Choroidal Excavation and } \\
\text { Leakage Area }\end{array}$ & $\begin{array}{c}\text { Choroidal } \\
\text { Hyperpermeability }\end{array}$ & $\begin{array}{c}\text { Punctate } \\
\text { Hyperfluorescent Spots }\end{array}$ & $\begin{array}{c}\text { Correlation between Focal Choroidal } \\
\text { Excavation and Choroidal } \\
\text { Hyperpermeability }\end{array}$ \\
\hline 2 & Yellowish lesion & Hyper & Adjacent to leakage area & Yes & Yes & Within Choroidal Hyperpermeability \\
\hline 3 & Pigmentary mottling & NA & Within leakage area & NA & NA & NA \\
\hline 5 & Pigmentary mottling & Hyper & Within leakage area & Yes & Yes & Within Choroidal Hyperpermeability \\
\hline 6 & Pigmentary mottling & Hypo & Within leakage area & Yes & Yes & Within Choroidal Hyperpermeability \\
\hline 7 & Yellowish lesion & Mixed & Within leakage area & Yes & Yes & Within Choroidal Hyperpermeability \\
\hline 8 & Pigmentary mottling & Mixed & Within leakage area & Yes & Yes & Within Choroidal Hyperpermeability \\
\hline
\end{tabular}

$\mathrm{NA}=$ Not available. ${ }^{\text {a Patient }} 4$ has bilateral focal choroidal excavation . 
Table 3. Eyes with and without Focal Choroidal Excavation Associated with Central Serous Chorioretinopathy

\begin{tabular}{|c|c|c|c|}
\hline & $\begin{array}{l}\text { CSC with Focal } \\
\text { Choroidal } \\
\text { Excavation }\end{array}$ & $\begin{array}{l}\text { CSC without } \\
\text { Focal Choroidal } \\
\text { Excavation }\end{array}$ & $P$ value \\
\hline $\mathrm{N}$ (eyes) & 9 & 107 & \\
\hline Sex (male/female) & $6 / 3$ & $92 / 15$ & $.144^{a}$ \\
\hline Age (years) & $54.9 \pm 13.6$ & $55.7 \pm 12.6$ & $.871^{b}$ \\
\hline Refractive error (diopters) & $-4.42 \pm 2.92$ & $-0.27 \pm 1.80$ & $.001^{b}$ \\
\hline Visual acuity (logMAR) & $-0.03 \pm 0.37$ & $0.07 \pm 0.28$ & $.731^{b}$ \\
\hline \multicolumn{4}{|l|}{ CSC Subtype, N (eyes) } \\
\hline Classic & $3(33.3 \%)$ & $53(49.5 \%)$ & \\
\hline Chronic & $6(67.7 \%)$ & $45(42.1 \%)$ & \\
\hline MPPE & 0 & $9(8.4 \%)$ & \\
\hline \multicolumn{4}{|l|}{ CSC Status, N (eyes) } \\
\hline Active & $4(44.4 \%)$ & $69(64.5 \%)$ & \\
\hline Resolved & $5(55.6 \%)$ & $38(35.5 \%)$ & \\
\hline Foveal retinal thickness $(\mu \mathrm{m})$ & $194.4 \pm 50.6$ & $270.8 \pm 108.7$ & $.040^{b}$ \\
\hline Foveal choroidal thickness $(\mu \mathrm{m})$ & $301.3 \pm 60.9$ & $376.6 \pm 104.8$ & $.036^{b}$ \\
\hline \multicolumn{4}{|c|}{${ }^{a}$ Fisher's exact test. ' Unpaired t-test. CSC = central serous chorioretinopathy; } \\
\hline \multicolumn{4}{|c|}{ LogMAR = logarithm of minimal angle of resolution. } \\
\hline
\end{tabular}


Click here to download high resolution image
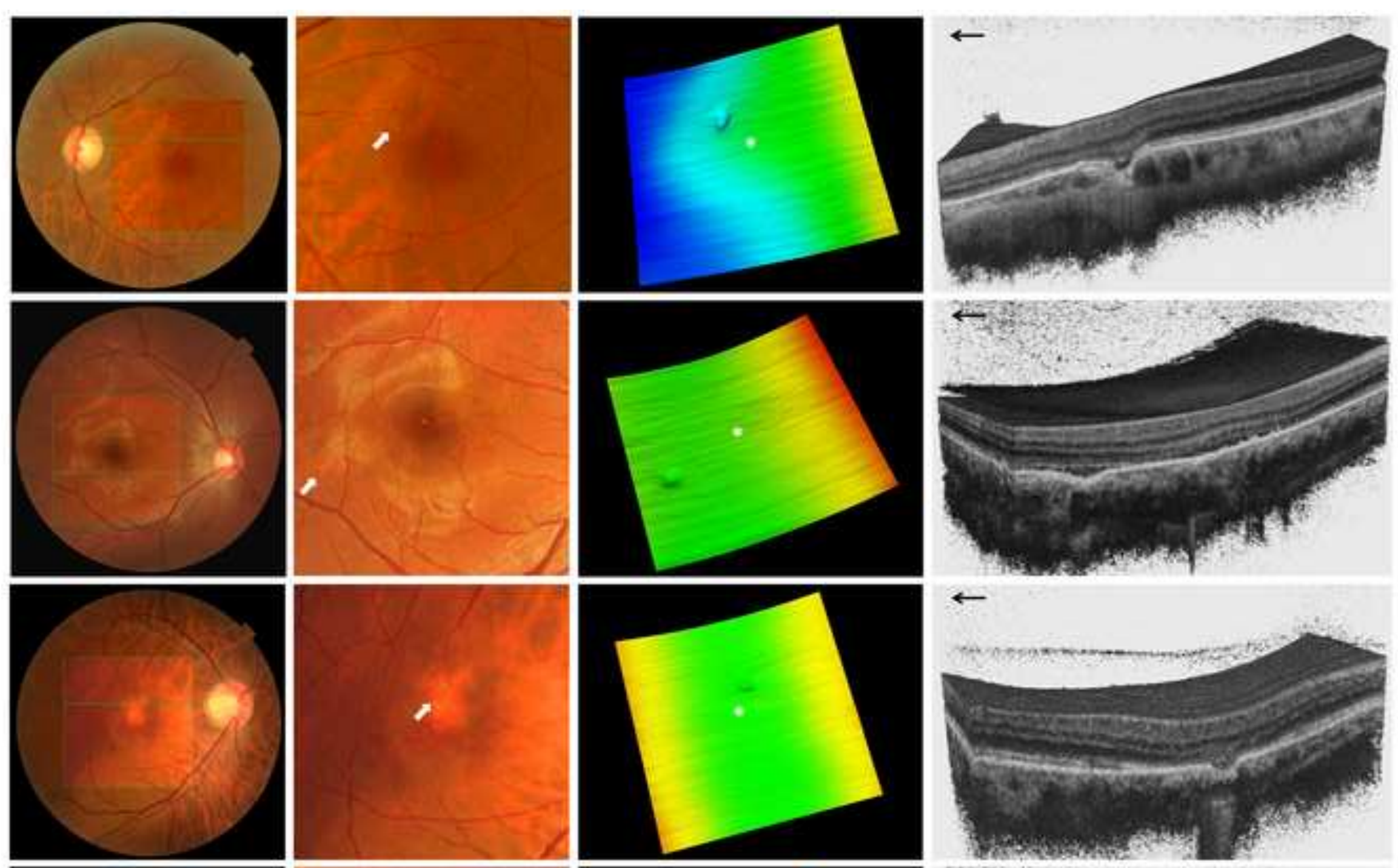

$\leftarrow$
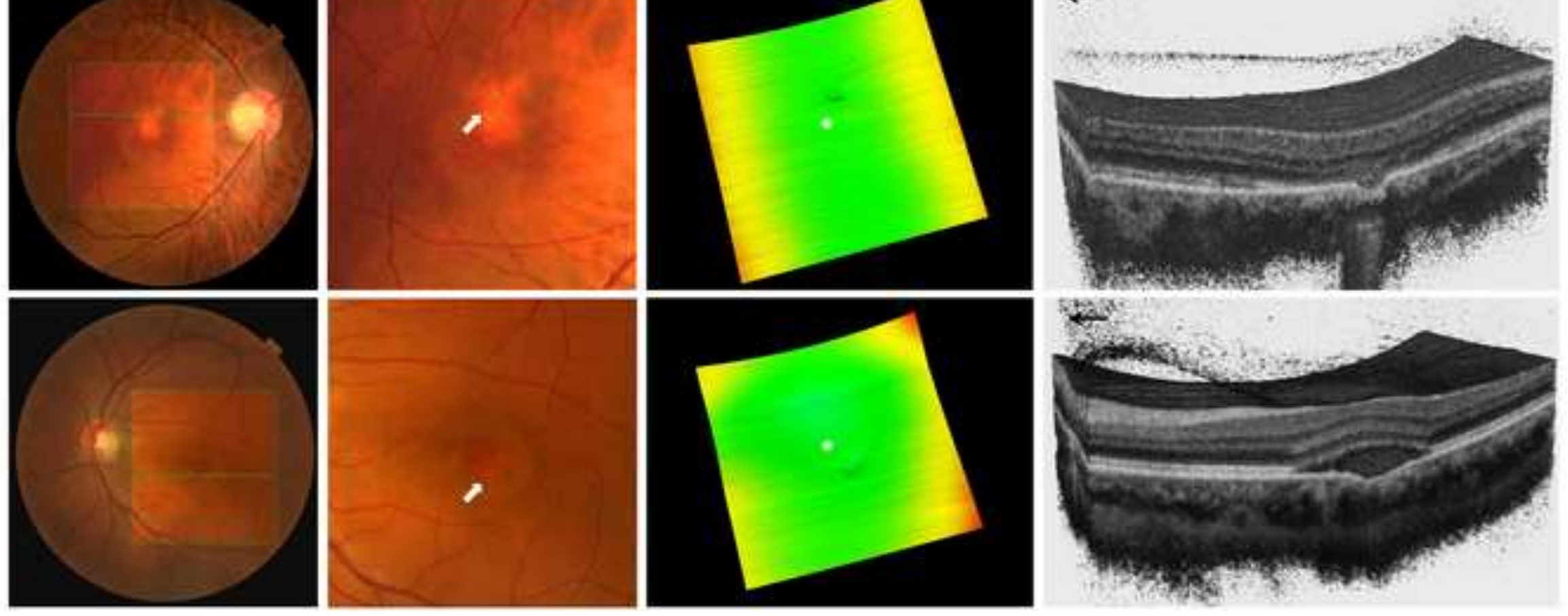
Click here to download high resolution image

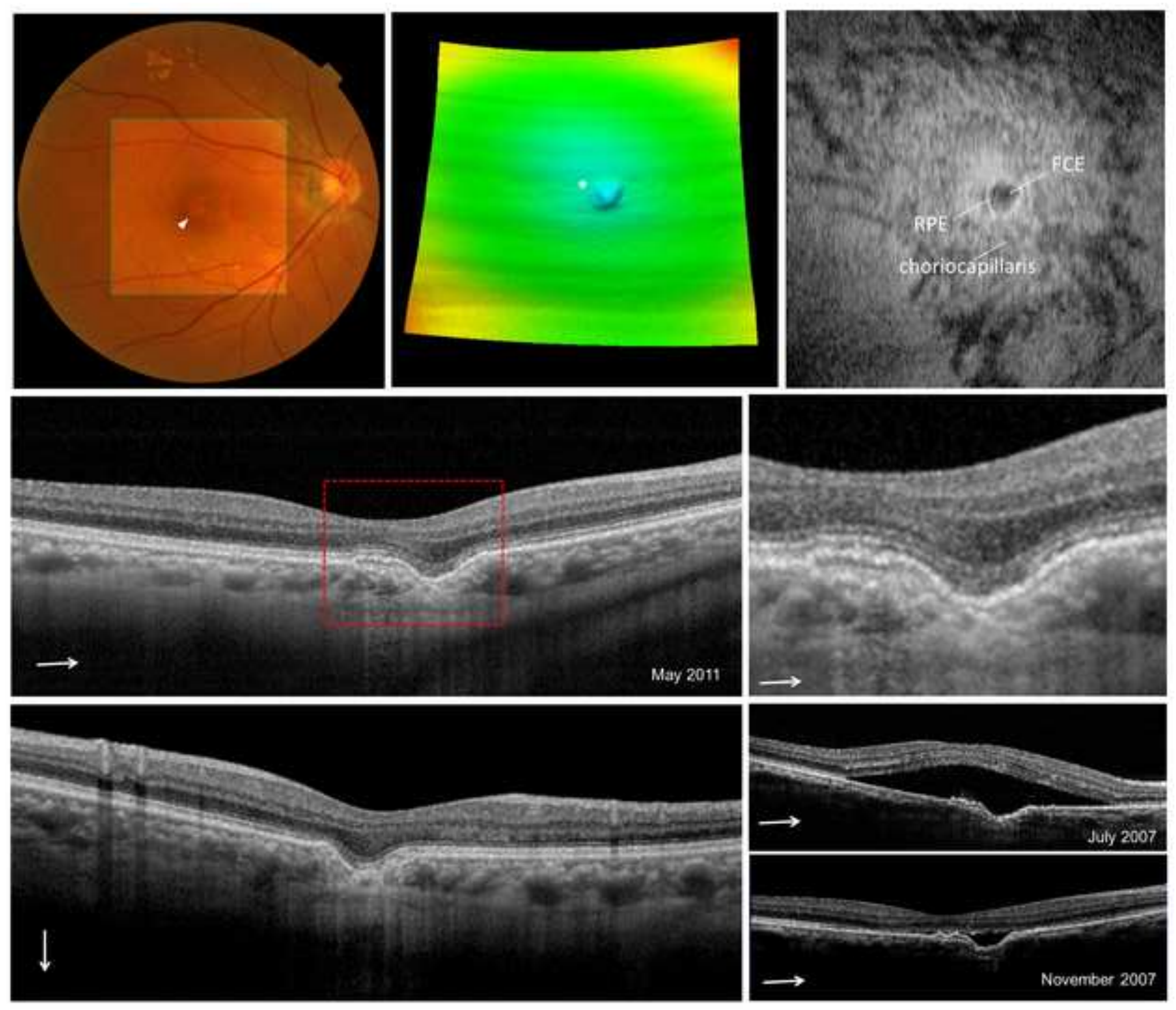





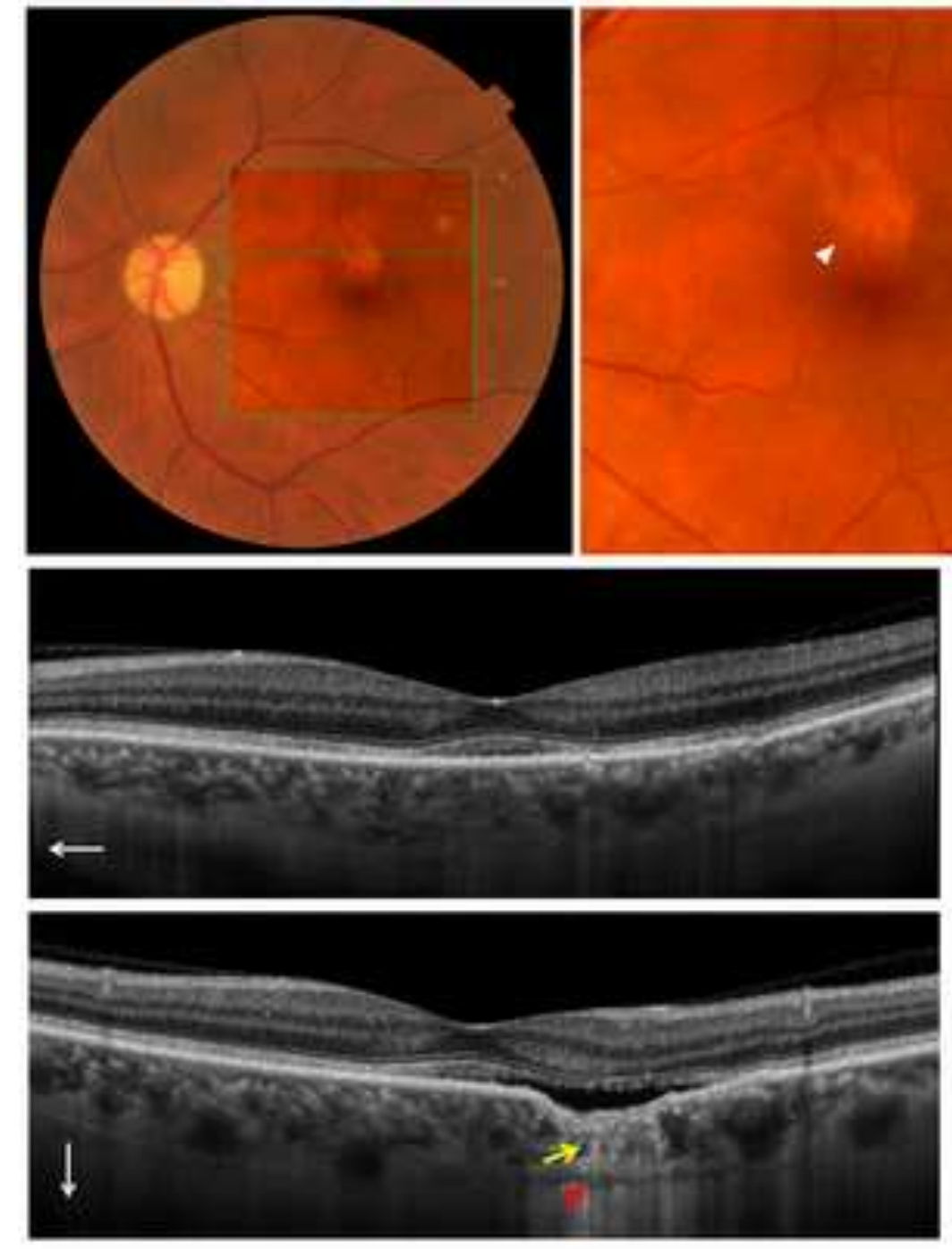
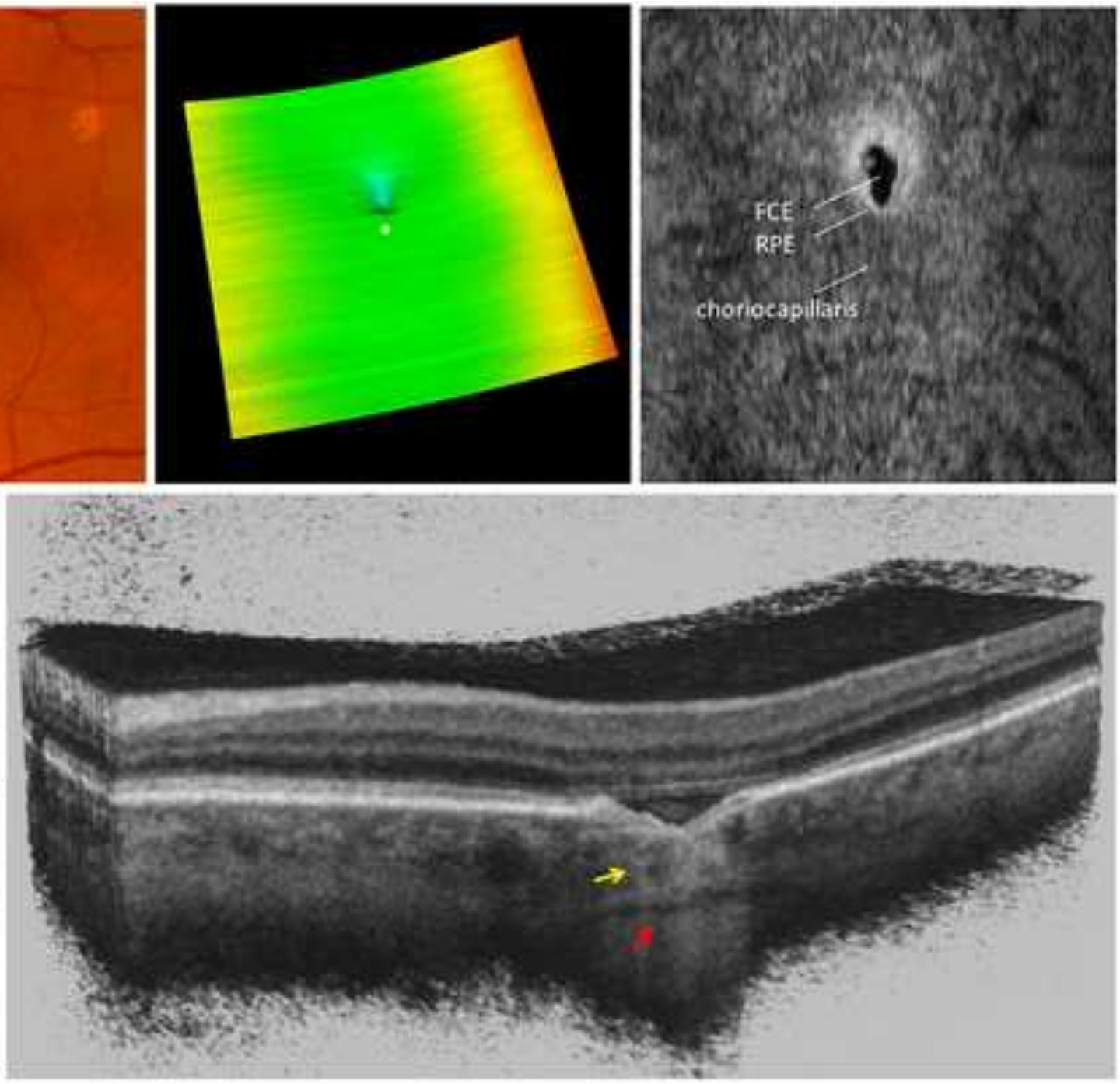






\section{Table of Contents Statement}

Focal choroidal excavations are seen in $7.8 \%$ of eyes with central serous

chorioretinopathy. Three-dimensional reconstructed optical coherence tomography

images allowed clear visualization of excavation morphology. Excavations were located

within both fluorescein leakage points and choroidal hyperpermeability areas. Focal

choroidal excavations may, in part, be involved in the pathogenesis of central serous

chorioretinopathy and can be formed by focal retraction of the retinal pigment epithelium

caused by scarring of the abnormal choroidal tissue. 